\title{
Research on Tower Crane Safety Control System Based on GPRS and U Disk Storage
}

\author{
Wang Jin-yu, a , Fu Guang-jie ${ }^{1, b}$, Lin Dong-xue ${ }^{1, c}$, Wang Chao ${ }^{1, d}$ \\ ${ }^{1}$ Northeast Petroleum University of Hei Long Jiang Province, China \\ a'wangjydxl@126.com, bfgjmhw@163.com, dwc1985@163.com
}

Keywords: sensor; data acquisition; GPRS; U disk storage

\begin{abstract}
Driven by the interests of the construction unit, field workers illegal operations and other reasons, tower crane collapse accidents occur frequently, personal injury or death became common occurrence. According to the national 《GB12602-2010 lifting mechanical overload protection device security technical specifications $》$, security and monitoring equipment must be installed on the tower crane. The GPRS and U disk storage tower crane control system has catered to the national security specified requirements. By the control system, the data can not only be recorded and inquired, well it can also be wireless remote transmitted. Supervisors who are in units can also monitor the construction site online. Meanwhile, the system can control the tower crane operations according to the specific situation of overrun. The system has a good promotional value.
\end{abstract}

\section{Introduction}

Traditional tower crane collapse accidents occur frequently because there is no installation of safety controller. The price is painful. Even if sometimes the tower crane did not collapse, but due to severe overweight tower is heavily skewed and balance arm bent also occurred, these bring great risks for the personal safety of the operating person. The same time as not installed tower crane controller, operating person could not accurately grasp the height of the heavy lifting, trolley location can not be precisely positioned, the rotation angle of tower crane not quite understand, the wind speed of working environment can also not accurately understand. These are not conforming to the provisions of the national 《GB12602-2010 lifting mechanical overload protection device security technical specifications》. There will be a great security risk. The supervising person of the project can't monitor in the unit. It's visible that tower crane monitoring system of real-time remote monitoring is the future development direction. And the technology of GPRS wireless access internet just solves this problem. U disk online storage provides convenience for querying tower crane accident reason.

\section{The Overall Structure And Control Process Of The Tower Crane Safety Control System}

Due to the limitations of traditional tower cranes control system. Tower crane control system based on GPRS and U disk storage can solve the above contradiction. The system hardware circuit and software circuit adopt modular thought. The overall structure is shown in Figure 1.

The system makes required signals convert to electric signals through the QS - 3 SSSF - A type weight sensor, SC - KG type height sensor, SSL type amplitude sensor, CQZE rotary sensors, SSF A type wind speed sensor. Use LM324 and AD620 to do the data processing. Through the MAX186CCPP we can realize A/D conversion. Through STC12C5A60S2 MCU we can achieve real-time data acquisition and Real-time display through the 7-inch LCD module. At the same time through the single chip microcomputer to store data in large capacity $U$ disk. And using macro electric HD7710, the GPRS module to implement data wireless remote transmission. According to the specification, preset upper and lower limits. If any overrun, drive the corresponding control relay, simulate the protection action. At the same time give out sound and light alarm. When a major incident, query and analysis results of the data can also be carried out by the large capacity U disk in the controller. The GPRS and U disk storage tower crane controller is well catered to the national 
《GB12602-2010 lifting mechanical overload protection device security technical specifications》 requirements.

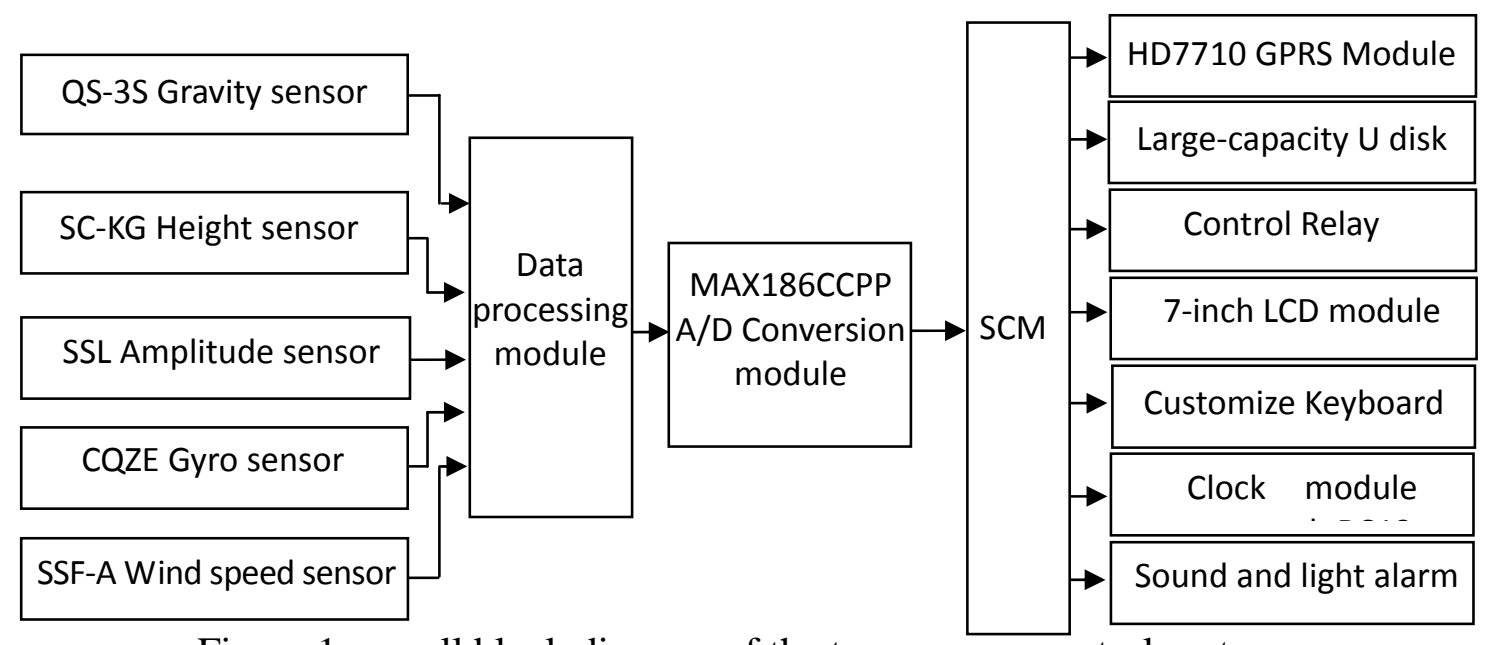

Figure 1 overall block diagram of the tower crane control system based on GPRS and U disk storage

\section{Core circuit design}

\section{Amplifier circuit of gravity small signal}

Due to the gravity signal changes in the range of $0 \sim 15 \mathrm{mV}$, it should be converted to $0 \sim 4.096 \mathrm{~V}$. The system uses the ideal integrated operational amplifier AD623 to amplify. Magnification is about 285 times. The specific circuit is shown in Figure 2:

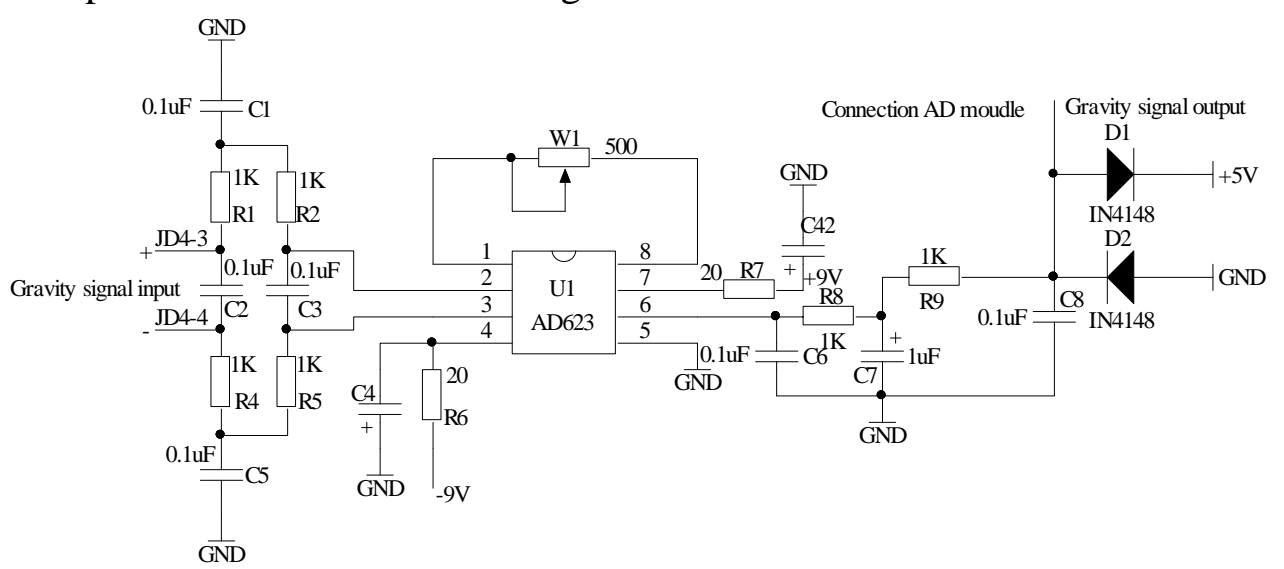

Figure 2 tower crane 12-bit data acquisition circuit design

The AD623 gain magnification is determined by the external resistor $\mathrm{W}_{1}$.

\section{Data Acquisition}

The accuracy of the data collection directly affects the precision of the control system. Because QS - 3 SSSF - A type gravity sensor voltage output ranges in $0 \sim 15 \mathrm{mV}$. The system uses the MAX186CCPP 12-bit AD for data acquisition. Precision can achieve 2 \%. Well meet the design technical requirements. The data converter collects gravity, rotary, height, amplitude and wind signals. The A/D conversion circuit is shown in figure 3. 


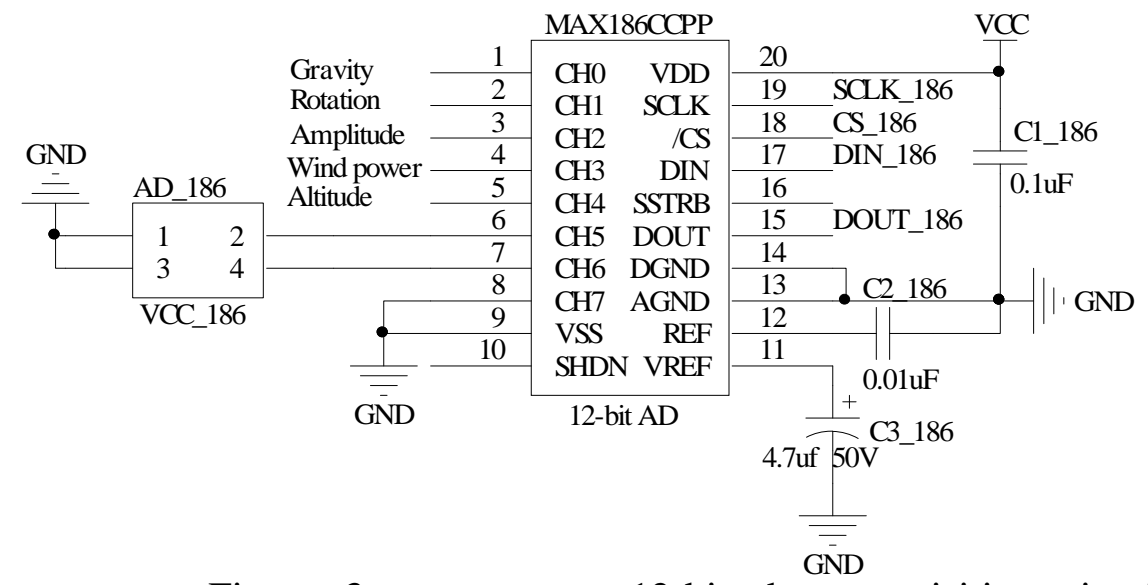

Figure 3 tower crane 12-bit data acquisition circuit

The conversion circuit uses Maxim's 12-bit A / D chip, 8-channel signal input. The input voltage requires in the range of 0 to $4.096 \mathrm{~V}$. Of course, the gravity signal requires op amp to amplify to standard signal before data being collected.

\section{Software design of the system}

The overall software design of the system should include several major modules. As follows, data collection, data processing, slave main program, control GPRS wireless data transmission, host the main program, the keyboard input, data display and alarm control. Among them, the data refers to the magnitude, torque, gravity, wind-speed, height and rotation angle when the tower crane is working. The software architecture is shown in Figure 4.

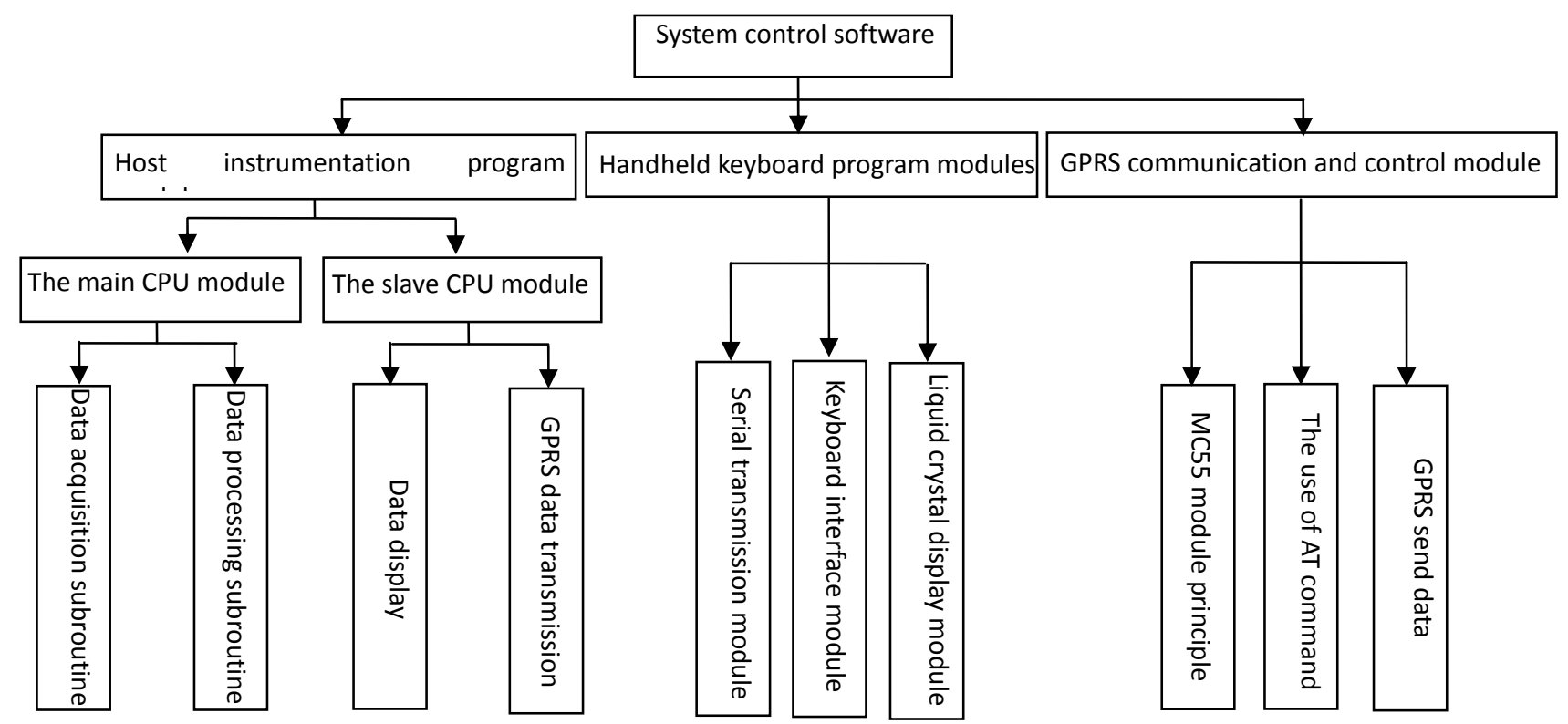

Figure 4 the architecture figure of the system software 
From the architecture figure, we can know that the terminal control software of the tower crane monitoring instrumentation is composed of three parts .They are the host instrument program modules, handheld keyboard program module, GPRS communication and control program modules. Each part of the software implementation and processing logic is elaborated in the following.

The PC software design of the system

The monitoring host with Double-CPU mode to realize data collection, processing, display and transmission functions. The main CPU completes the data collection, processing and relay operation, etc. The slave CPU completes the display and GPRS transmission of data. Since signals are slowly varying signals, the interval of acquisition time can be a few seconds. The flow chart is shown in Figure 5.

The main CPU completes a collection of data then transmits to the slave, the latter refresh the display driver chip. The

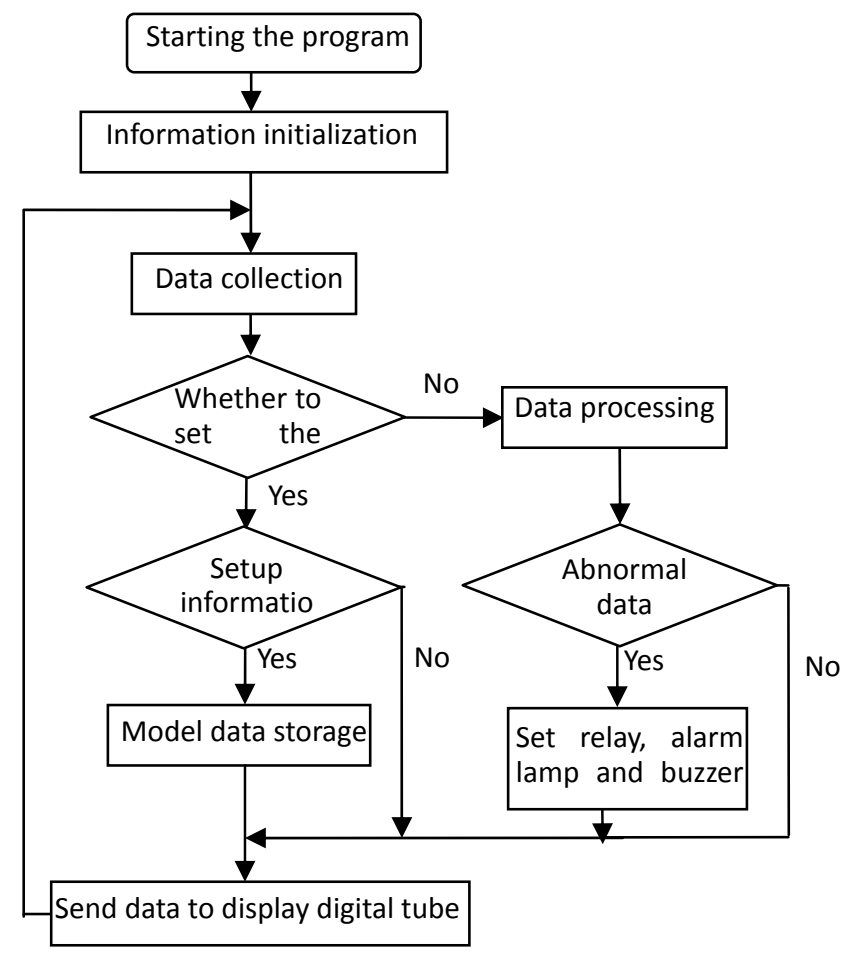

Figure 5 the software flowchart of the main program data includes monitoring data and time information. The master program of the system host is the main control software for the entire system operating normally. And it calls the other module to implement the communication and data display between the host and the various sub-machines.

\section{The monitoring software design of the tower crane}

The tower crane monitoring software is user-oriented. Therefore, it has to pay special attention to friendly operation interface. Simple used. Good stability and cross-platform. Meet the requirements of the user running on a variety of operating systems and a variety of PC. Monitoring software is divided into the interface module, communication module and database management module. The framework of the system interface is shown in Figure 6.

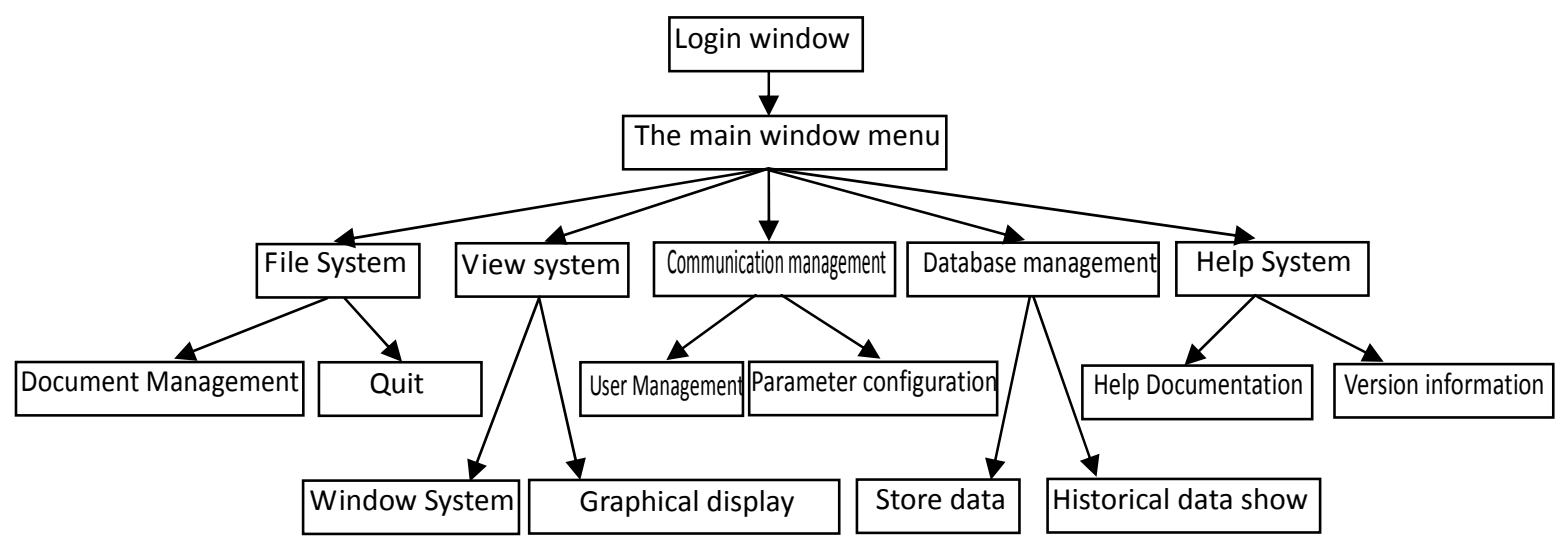

Figure 6 the interface framework of the tower crane monitoring software

Through good monitoring interface, users can easily understand the operational status of the tower crane. Better dynamic monitoring of the tower crane, greatly reduce the accident probability.

\section{Summary}

The installation of the tower crane controller can alarm and control for irregularities. Greatly 
improve the safety of the operating person. Well catered to the national 《GB12602-2010 lifting mechanical overload protection device security technical specifications》 requirements. The data can be displayed on a 7-inch LCD display in real time. So to the operating person, it can facilitate the next operation and understand the current state of the tower crane. These provide a reliable guarantee for the operator to achieve the correct operation. Since the controller have internal HD7710 wireless GPRS module. Supervisors who in the units can monitor the construction site online. Don't have to go to the scene to supervise. This will save time and effort .It can give great convenience for project supervision. Because the controller has the large capacity U disk which the data are stored in. Data can be queried and analysis in situ. This can facilitate accident analysis. The controller uses the MAX186CCPP 12-bit AD for data acquisition. Precision can achieve 2 \%. So the accuracy of data collection is greatly improved.

\section{References}

[1] Deng Li, Lin Hua, Luo Yan, Tian Jian-guo, Yu bo, Xi De-miao. With the problem of identification of tower cranes explore [J]. Building Science Research of Sichuan . 2009(03)

[2] Cao Ling-zhi, Wang Gong-wei, Li Chun-en, Chen Hua-ying, Miao Wei-pu. Status and trends of the crane VVVF control technology [J]. Micro-motor . 2008(03)

[3] Shao Guo-ping. Tower crane safety monitoring and recording system developed [J]. Heilongjiang Science and Technology Information . 2009(33)

[4] Mi Xin-Jiang, Ren Jian-qiang. New tower crane smart torque measurement and control instrument development [J]. Instrumentation technology and sensors. 2010 (03)

[5] Huang qun. The defective acoustic emission signals of the crane girder activity characteristics [J]. Petroleum and Chemical Equipment 2012 (06)

[6] WU Jian-qi. Tower crane slewing broken shaft of the motor and countermeasures [J] Wireless Internet Technology 2012 (06)

[7] Yan bin. Importance of the tower crane safety protection device [J]. China Science and Technology Information 2012 (11)

[8] Yu Shu-hong, Liu Zhan-sheng, Yu Qun. Building tower crane fault monitoring system based on C8051F[J]. Construction machinery. 2008(17) 